\title{
Phononic band gap and wave propagation on multiferroic-based acoustic metamaterials
}

\author{
Selami Palaz ${ }^{\mathrm{a}}$, Zafer Ozer ${ }^{\mathrm{b}}$, Oral Oltulu ${ }^{\mathrm{a}}$, Amirullah M. Mamedov ${ }^{\mathrm{c}, \mathrm{d}}$, and E. Ozbay ${ }^{\mathrm{c}}$ \\ ${ }^{a}$ Department of Physics, Harran University, Sanliurfa, Turkey; ${ }^{\text {b}}$ Electronic Technology, Mersin Vocational \\ School, Mersin University, 33335, Mersin, Turkey; ${ }^{C}$ Nanotechnology Research Center (NANOTAM), Bilkent

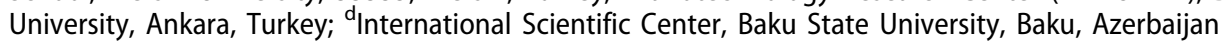

\begin{abstract}
In the present work, the acoustic band structure of a two-dimensional (2D) phononic crystal containing a multiferroic and liquid were investigated by the plane-wave-expansion method. 2D PnC with triangular and honeycomb lattices composed of $\mathrm{LiCu}_{2} \mathrm{O}_{4}$ cylindrical rods embedded in the seawater matrix are studied to find the existence of stop bands for the waves of certain energy. Phononic band diagram $\omega=\omega(\mathbf{k})$ for a 2D PC, in which nondimensional frequencies $\omega \mathrm{a} / 2 \pi \mathrm{c}$ (c-velocity of wave) were plotted versus the wavevector $\mathbf{k}$ along the $\Gamma-X-\mathrm{M}-\Gamma$ path in the Brillouin zone show few stop bands in the frequency range between 10 and $110 \mathrm{kHz}$.
\end{abstract}

\section{ARTICLE HISTORY}

Received 14 May 2018 Accepted 31 October 2018

\section{KEYWORDS}

Multiferroic; phononic crystal; metamaterial; equifrequency surface; group velocity

\section{Introduction}

Phononic crystals (PnCs) and acoustic metamaterials have generated rising scientific interests for very diverse technological applications ranging from sound abatement to ultrasonic imaging to telecommunications to thermal management and thermoelectricity [1]. PnCs and acoustic metamaterials are artificially structured composite materials that enable the manipulation of the dispersive properties of vibrational waves. Finally, the study of PnCs and acoustic metamaterials has also extensively relied on a combination of theory and experiments that have shown extraordinary complementarity [2]. Due to their interesting property, the phononic structures have extensive practical applications by suppressing sound waves for a given frequency range. Therefore, the application of PnCs can be mainly determined by manipulating the regulation performance level of the band gap. The condition of controlling and tuning band gap in conventional research is primarily met by changing the geometry of lattice, filling fraction and orientation of scatterers in a $\mathrm{PnC}[3,4]$. Characteristics of PnCs can be altered by changing the geometry of the inclusions or by varying the elastic characteristics of the constitutive materials. Therefore, different materials are needed to fabricate phononic structures to obtain reachable frequencies for the energy band gaps from about kilohertz to megahertz range and even to ultra- high frequencies (very high-frequency sound, with $\mathrm{f}<1 \mathrm{GHz}$ ) [5-10]. For our calculations we have considered the case of the propagation of a longitudinal wave in the phononic crystal structure. 
(a)

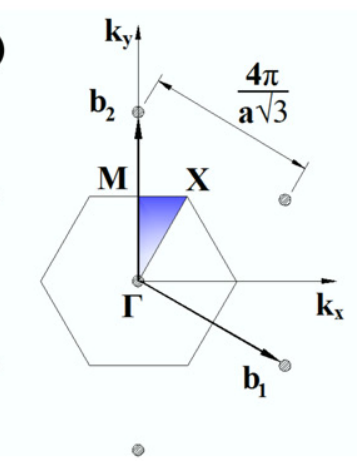

(b)

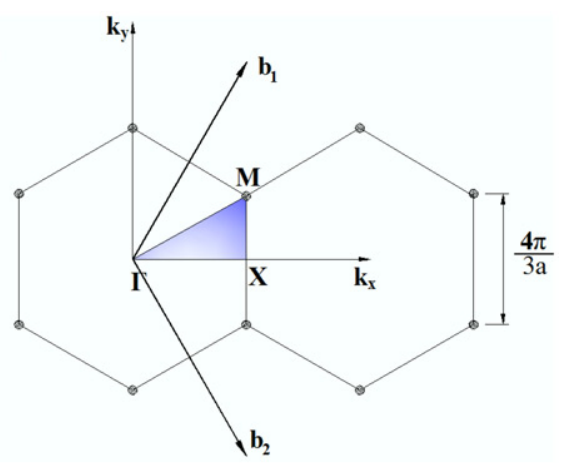

Figure 1. Brillouin zone of (a) the triangular lattice and (b) the honeycomb lattice.

\section{Model and method of calculation}

The wave propagation in a homogeneous medium can be strongly changed by inserting periodical inclusions with different elastic constants. The periodic arrangement in these so-called PnCs induces a wave scattering and destructive interferences can appear in some frequency ranges, leading to forbidden band-gaps. The system in our study was modeled as solid-fluid phononic crystal. We consider a $2 \mathrm{D}$ system consisting of infinitely long cylinders aligned along the $\mathrm{z}$ direction and any material based parameters are independent of the $z$ direction. The phononic crystal also extends infinitely in the $x y$ plane. Therefore, the mechanical properties of the phononic crystal vary periodically in the $x y$ plane. In this study we only consider the longitudinal polarization and, therefore, the propagation of the acoustic wave is assumed to be only in the $x y$ plane. For software implementation and calculation the code written by Elford was used [11]. In this work, we adopted the COMSOL Multiphysics, a commercial package based on the finite-element method (FEM).

\section{Results and discussion}

The numerical calculation in this study has the parameters with the column radius of $16.5 \mathrm{~mm}$ and a lattice size of $\mathrm{a}=\mathrm{b}=33 \mathrm{~mm}$ for an array of circular $\mathrm{LiCu}_{2} \mathrm{O}_{4}$ cylinders embedded in an liquid background (filling ratio change from $30 \%$ up to $70 \%$ ). The phononic material parameters used in the calculations are $E=156.4 \mathrm{GPa}$ (Young modulus), $\rho_{\mathrm{LiCu} 2 \mathrm{O} 4}=6.29 \mathrm{~kg} / \mathrm{m}^{3}$ and $\rho_{\text {seawater }}=3.67 \mathrm{~kg} / \mathrm{m}^{3}$, sound velocity $(\mathrm{C})$ are $\mathrm{CLiCu}_{2 \mathrm{O} 4}=6690$ $\mathrm{m} / \mathrm{s}$ and $C_{\text {seawater }}=1560 \mathrm{~m} / \mathrm{s}$. PnCs with triangular and hexagonal lattices are considered. These lattices consist of $\mathrm{LiCu}_{2} \mathrm{O}_{4}$ circular cylinders placed in seawater forming twodimensional lattices with lattice spacing a. Figu. $1(\mathrm{a}, \mathrm{b})$ is the Brillouin regions of the triangular lattice and the hexagonal lattice, respectively. The irreducible part of the Brillouin zone of a triangular lattice is shown in Fig. 1(a) which is a triangle with vertices $\Gamma, \mathrm{X}, \mathrm{M}$. The irreducible part of the Brillouin zone of a honeycomb lattice is shown in Fig. 1(b), which is a triangle with vertices $\Gamma, X, M$.

In order to show the feature appearing in the band structures of the proposed structure (honeycomb), we illustrate the dispersion curves for a typical circular hole with an $r / a=0.5$ in Fig. 2 that corresponds to the maximum filling ratio. The plots were given 


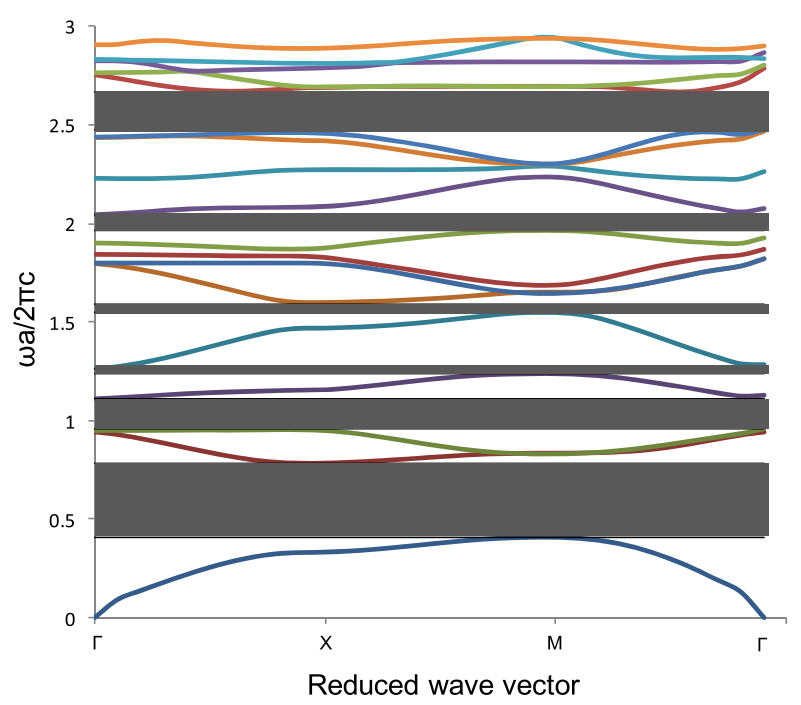

Figure 2. Acoustic band structures for solid $\mathrm{LiCu}_{2} \mathrm{O}_{4}$ rods in the seawater host.

in terms of the reduced frequency $\omega a / 2 \pi c_{l}$, which is the vertical axis and the horizontal axis is the reduced wave vector. All of the parameters are independent of the $z$ direction. That means the $2 \mathrm{D} \mathrm{PnC}$ structure is invariant along the $z$ direction. The first eighteen bands for propagation in the $(x, y)$ plane were computed while the irreducible triangle $\Gamma-\mathrm{X}-\mathrm{M}-\Gamma$ of the Brillouin zone along its periphery was being scanned. For band structure calculations, moving along the high symmetry axes of the first Brillouin zone was good enough. Fig. 2 shows full band gaps along the $\Gamma-\mathrm{X}-\mathrm{M}-\Gamma$ direction for honeycomb lattice. First (0.4054-0.7812), second (0.9575-1.2411), third (1.2411-1.2611), fourth (1.5503-1.5901), fifth (1.9665-2.0478), and sixth (2.4747-2.6705).

Then we investigated the case with composite rods placed in the seawater in triangular lattice with a circular cross section. For the triangular lattice, the largest absolute phononic stop band is produced when the filling fraction $f=0.58$, the maximum gap (between the 2nd and 3rd bands) has the largest width (gap-midgap ratio) $\Delta \omega / \omega g=0.1355$.

It is known that for a wave propagating in an isotropic medium with a fixed frequency $\omega$, the equi-frequency surface represents a sphere. In this case, the wave vector $\vec{k}$ and group velocity vector $\vec{V}_{g}$, which determines the ray direction, are always parallel. However, the equi-frequency surface is not spherical for anisotropic media and the vectors $\vec{V}_{g}$ and $\overrightarrow{\boldsymbol{k}}$ are not parallel $[12,13]$ By analogy with 3D case, the propagation, reflection, and refraction of the wave in $2 \mathrm{D}$ structure can be described in terms of the equifrequency dependence that can be considered as the section of the dispersion surface $\omega\left(k_{x}, k_{y}\right)$ in the space of variables $\left\{\omega, k_{x}, k_{y}\right\}$ by the plane corresponding to constant frequency. It is well known that the analysis of equi-frequency dependences is the most efficient in the studies of 2D geometries, especially in solving problems when only orientations of the $\vec{V}_{g}$ and $\overrightarrow{\boldsymbol{k}}$ vector of incident, reflected, and refracted waves are of interest, and are not the amplitudes of the reflected and refracted rays. The equi-frequency dependence has a simple physical meaning for the analysis of $2 \mathrm{D}$ geometries: since this dependence describes all the possible waves with the given frequency $\omega$ and 

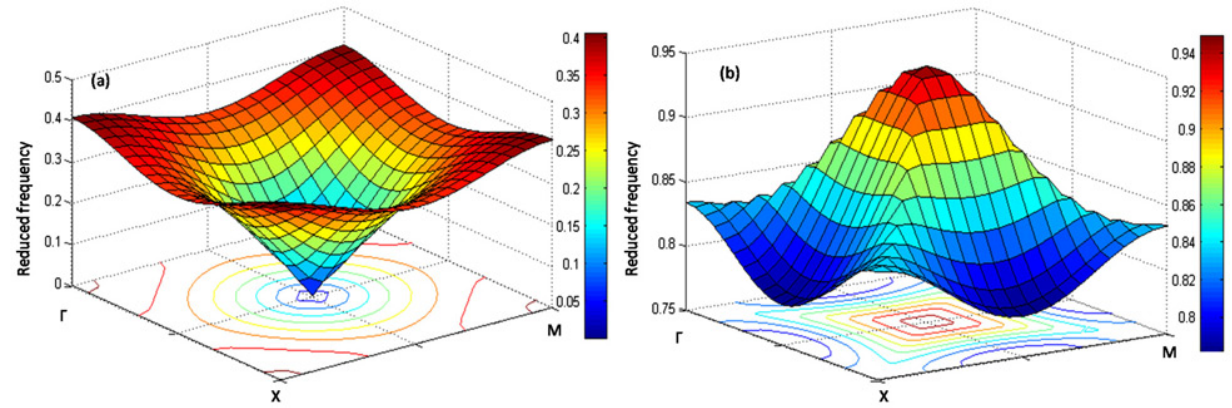

Figure 3. 3D dispersion curves and equifrequency contours for the first (a) and for the second (b) modes.

various wave vectors, the directions of the reflected and the refracted rays can be determined by simply finding the points in equi-frequency dependences of media that satisfy the momentum conservation law at a known orientation of the boundary and a given angle of incidence of the wave. Now, we present some numerical examples for our PnC structures. In all of these examples, we exploit symmetry to calculate the equi-frequency surfaces over the irreducible Brillouin zone of the entire Brillouin zone. First, we consider the equi-frequency surface of a honeycomb lattice of $\mathrm{LiCu}_{2} \mathrm{O}_{4}$ layers in seawater. Here, the map was discretized using 441 points per edge of the unit cell for the first band in Fig. 3(a). The map was discretized using 441 points per edge of the unit cell for the next band in Fig. 3. The curves shown correspond to equi-frequency surfaces of the lowest order band up to frequencies just below the band gap starting at around $0.4(2 \pi \mathrm{c} / \mathrm{a})$. For the next band, the curves shown correspond to equi-frequency surfaces of the lowest order band up to frequencies just below the band starting at around $0.78(2 \pi \mathrm{c} / \mathrm{a})$.

A pulse wave propagating along the PC let the pulse be finite in dimension along the direction of propagation. Theoretically, such a pulse can be represented as a wave packet formed as a superposition of the modes, but with a different propagation constant [12]. From [12-13], it follows that the envelope of the wave packet propagates with the velocity $V_{g}^{z}=\omega_{n}(\beta)$, where $\beta$ is propagation constant. The direct calculation of the derivative of the dispersion relation calculated numerically is not always convenient and can give an error. The group velocity of the wave packet $V_{g}^{z}$, is equal to the velocity of energy transfer by the mode $n \beta$. Thus, by using these results the group velocity can always be calculated with more accuracy, irrespective of the number of points in the dispersion curve with $\mathrm{LiCu}_{2} \mathrm{O}_{4}$ layers in a seawater background. Fig. 3 shows the dispersion curves for the lowest and second mode with their associated reduced velocities along the $\mathrm{X}-\Gamma-\mathrm{X}$ direction in (a) and (b), respectively. The dependences $V_{g}^{z}(\beta)$, which describe the wave packets of localized modes of any polarization and any order, exhibit (generally) a maximum at certain propagation constants.

\section{Conclusion}

In this paper, the band gap properties of a honeycomb and triangular lattices phononic crystals consisting of multiferroic $\mathrm{LiCu}_{2} \mathrm{O}_{4}$ cylindrical rods in seawater were investigated 
by FEM calculation. We considered a new design of $\mathrm{LiCu}_{2} \mathrm{O}_{4}$ metamaterial to study wave propagation. A series of numerical simulation for the full waveforms of elastic waves was performed in a frequency domain in order to investigate the band gap characteristics of proposed PnC structure. The modal behavior was utilized to understand the elastic wave propagation in a honeycomb and triangular lattices configurations. It is found that significant band gaps are available at low frequencies. The appearance of these absolute bands at low frequencies is due to the complexity of the medium. It must be noted that the filling ratio for the composite system is not small and therefore for such a filling ratio, noticeable pass-bands were obtained at low frequencies.

\section{Acknowledgments}

This work is supported by the projects DPT-HAMIT, DPT-FOTON, One of the authors (Ekmel Ozbay) also acknowledges partial support from the Turkish Academy of Sciences.

\section{References}

[1] Y. Pennec, et al. Phononic crystals and manipulation of sound. Phys. Status Solidi. C6, No.9, 2080-2085 (2009). DOI: 10.1002/pssc.200881760.

[2] Y. Tanaka, S. I. Tamura, Surface acoustic waves in two-dimensional periodic elastic structures. Physical Review B.; 58, 7958 (1998). DOI: 10.1103/PhysRevB.58.7958.

[3] W. M. Kuang, et al. The bandgap of a photonic crystal with triangular dielectric rods in a honeycomb lattice. J. Opt. A-Pure Appl. Opt. 7, 525 (2005). DOI: 10.1088/1464-4258/7/10/001.

[4] R. Z. Wang, et al. Effects of shapes and orientations of scatterers and lattice symmetries on the photonic band gap in two-dimensional photonic crystals. J. Appl. Phys. 90, 4307(2001). DOI: 10.1063/1.1406965.

[5] R. H. Olson and I. El-Kady, Microfabricated phononic crystal devices and applications, Measur. Sci. Technol. 20, 012002 (2009). DOI: 10.1088/0957-0233/20/1/012002.

[6] H. Pichard, et al. Two-dimensional discrete granular phononic crystal for shear wave control. Phys. Rev. B. 86, 134307 (2012).

[7] P. M. Walker, et al. Coherent elastic waves in a one-dimensional polymer hypersonic crystal. Appl. Phys. Lett. 97, 073106 (2010). DOI: 10.1063/1.3479929.

[8] P. A. Deymier, et al. Rotational modes in a phononic crystal with fermion-like behavior. J. Appl. Phys. 115, 163510 (2014). DOI: 10.1063/1.4872142.

[9] M. Sigalas, and E. N. Economou, Band Structure of Elastic Waves in Two Dimensional Systems. Solid State Commun. 141, 86 (1993). DOI: 10.1016/0038-1098(93)90888-T.

[10] T. Gorishnyy, Hypersonic phononic crystals, PhD thesis, Department of Materials Science and Engineering at the Massachusetts Institute of Technology, 2007.

[11] D. P. Elford, Band gap formation in acoustically resonant phononic crystals, $\mathrm{PhD}$ thesis, Loughborough University Institutional Repository, 2010.

[12] E. H. Lock, The properties of isofrequency dependences and the laws of geometrical optics. Physics - Uspekhi. 51 (4), 375 (2008).

[13] A. V. Kildishev, and V. M. Shalaev, Transformation optics and metamaterials. Physics-Uspekhi. 54 (1), 53 (2011).

[14] M. S. Kushwaha, et al. Acoustic band structure of periodic elastic composites. Phys. Rev. Lett. 71, 2022 (1993). DOI: 10.1103/PhysRevLett.71.2022. 